ISSN 1991-8631

Original Paper

http://indexmedicus.afro.who.int

\title{
Relation entre les anomalies du spermogramme et les constituants biochimiques du liquide séminal de sujets consultant pour hypofertilité masculine à Ouagadougou
}

\author{
Jean SAKANDÉ*, Elie KABRÉ, Axelle EKUE-LIGAN, Hugues Aristide OUÉDRAOGO \\ et Mamadou SAWADOGO
}

\author{
Laboratoire de Biochimie UFR Sciences de la Santé de Ouagadougou et Laboratoire de Biochimie du CHU \\ Yalgado Ouédraogo, Burkina Faso. \\ *Auteur correspondant; E-mail: jsakande@gmail.com ; 09 BP 863 Ouagadougou 09.
}

\section{RESUME}

La présente étude visait à analyser le profil biochimique du liquide séminal de patients consultant pour hypofertilité masculine à Ouagadougou, en relation avec les anomalies du spermogramme. La population d'étude était constituée de 220 sujets masculins âgés de 23 à 64 ans. Les dosages du glucose, du cholestérol, des triglycérides, des protéines totales, du fructose, du calcium, du magnésium, du zinc et de l' $\alpha$-glucosidase ont été réalisés avec un automate Cobas Mira (Roche) alors que le sodium et le potassium ont été dosés avec un photomètre de flamme (PHF 104 de Hycel). L'électrophorèse a été réalisée sur support hydragel avec une chaîne d'électrophorèse Hydrasis (Sébia). L'analyse du spermogramme a montré que seuls 35 sujets soit 15,9\% avaient un spermogramme normal contre $84,1 \%$ d'anomalies. La répartition des anomalies du spermogramme observées était : asthénospermie (25\%), oligo-asthénospermie (15,5\%), oligo-asthéno-tératospermie (13,6\%), azoospermie (10,5\%), oligospermie $(10,5 \%)$, tératospermie $(9,1 \%)$. Le profil électrophorétique des protéines du liquide séminal n'a pas apporté un intérêt notable dans l'étude des différentes anomalies du spermogramme. Cependant, l'augmentation du sodium dans l'azoospermie, la diminution du magnésium et l'augmentation des triglycérides dans l'asthénospermie et la diminution du potassium dans l'oligospermie présenteraient un intérêt s'ils étaient confirmés par d'autres travaux. En effet, ces paramètres de réalisation courante dans la plupart des laboratoires africains, pourraient être utiles dans l'exploration de l'hypofertilité masculine.

(c) 2012 International Formulae Group. All rights reserved.

Mots clés: sperme, profil biochimique, anomalies morphologiques, infertilité.

\section{INTRODUCTION}

Les problèmes d'infertilité touchent environ un couple sur 10 dans le monde (Beaudeux et Durand, 2011). La fréquence de l'hypofertilité masculine isolée varie de 10$26 \%$ en France (AFSSAPS, 2004). Au Burkina Faso, la stérilité masculine est estimée à environ 30\% de l'hypofertilité du couple (EDSBF, 2003). C'est dire la part prépondérante de l'homme qui pendant longtemps, dans notre contexte, a indexé la femme sans défense comme l'unique responsable de la stérilité du couple. 
L'exploration de la fertilité chez l'homme est basée d'abord sur la réalisation d'un spermogramme après 2 à 3 jours d'abstinence (WHO, 2010). En cas d'anomalies du spermogramme, un caryotype et des dosages hormonaux pourront être envisagés et certains marqueurs biochimiques du liquide séminal sont habituellement dosés. Les constituants biochimiques du sperme ont des origines spécifiques leur conférant le rôle de marqueurs spécifiques des sécrétions des glandes génitales mâles (Beaudeux et Durand, 2011). Chacun des segments des voies génitales fabrique une molécule qui lui est propre et qui passe dans la phase liquide du sperme, appelée liquide séminal : l'épididyme fabrique de l' $\alpha$-glucosidase et de la carnitine, les vésicules séminales fabriquent du fructose et la prostate de l'acide citrique et du zinc. Les variations de concentration de ces marqueurs peuvent confirmer ou orienter vers une lésion anatomique ou un dysfonctionnement d'un des organes de l'appareil génital masculin à l'origine d'une hypofertilité (Delattre et al., 2003). Ces dosages du liquide séminal sont rarement proposés dans les services de santé du fait du manque de laboratoire de biologie spécialisé, de la faiblesse de la demande de ces analyses et surtout du coût inaccessible. La présente étude visait à analyser le profil biochimique $\mathrm{du}$ liquide séminal des patients consultant pour hypofertilité masculine à Ouagadougou en relation avec les anomalies du spermogramme en y associant les paramètres biochimiques courant plus accessibles en vue de rechercher des marqueurs biochimiques alternatifs pouvant servir à la prise en charge de l'hypofertilité masculine dans notre contexte.

\section{MATERIEL ET METHODES}

\section{Cadre de l'étude et type d'étude}

Il s'agit d'une étude descriptive transversale qui s'est déroulée à Ouagadougou au Burkina Faso de janvier à juillet 2008. Du fait que le spermogramme n'est pas pratiqué dans le secteur public, les sites de prélèvement étaient constitués des trois principaux laboratoires privés de la ville à savoir, le laboratoire du Centre, le laboratoire Sainte Elizabeth et le laboratoire de la clinique Philadelphie.

Les analyses biochimiques ont été réalisées au Laboratoire de Biochimie du Centre Hospitalier Universitaire Yalgado Ouédraogo et au Laboratoire de Biochimie de l'Unité de Formation et de Recherche en Sciences de la Santé de l'Université de Ouagadougou.

\section{Population d'étude}

La population d'étude était constituée de 220 sujets consultant pour hypofertilité. Ont été inclus dans l'étude, les sujets consultant pour hypofertilité et ayant eu à faire un spermogramme dans l'un des laboratoires retenus pour l'étude. Tous les participants ont donné leur consentement éclairé après explication de l'intérêt de l'étude.

\section{Méthodes}

Les paramètres étudiés sont: le spermogramme, les dosages biochimiques spécifiques du sperme (l' $\alpha$-glucosidase, le fructose, le zinc) auxquels nous avons adjoint des paramètres moins habituels mais faciles à réaliser dans la plupart des laboratoires du pays (glucose, cholestérol, triglycéride, protéines totales, magnésium, calcium, sodium, potassium et électrophorèse des protéines).

\section{Le spermogramme}

Le spermogramme a été immédiatement réalisé sur du sperme recueilli au laboratoire par masturbation dans un flacon à large goulot stérile chez des sujets ayant observé une abstinence de 3 à 5 jours selon le protocole OMS (2010). Le liquide séminal a été séparé 
par centrifugation à $3000 \mathrm{~g}$ pendant 15 minutes après le temps de liquéfaction. Ce plasma séminal a été conservé à $4{ }^{\circ} \mathrm{C}$ jusqu'à l'analyse biochimique. Les normes OMS (2010) consignées dans le Tableau 1 ont permis de classer les différents spermogrammes.

\section{Les paramètres biochimiques}

Les dosages du glucose, du cholestérol, des triglycérides, des protéines totales, du fructose, du calcium, du magnésium, du zinc et de l' $\alpha$-glucosidase ont été réalisés avec un automate Cobas Mira (Roche) alors que le sodium et le potassium ont été dosés avec un photomètre de flamme (PHF 104 de Hycel).
Le Tableau 2 présente les principes de dosage des paramètres biochimiques. L'électrophorèse a été réalisée sur support hydragel avec une chaîne d'électrophorèse Hydrasis (Sébia).
Analyse statistique
Le traitement des données a été fait à l'aide du logiciel Epi-info version 6.04.fr. Le test " $t$ " de Student a permis de comparer les différentes moyennes et le calcul de coefficients de corrélation a permis de rechercher les liaisons entre les différents paramètres. Le seuil de signification retenu était $\mathrm{p}<0,05$.

Tableau 1: Critères de définition des anomalies du sperme selon l'OMS (2010).

\begin{tabular}{|c|c|c|}
\hline Normes OMS & Définitions de l'anomalie & $\begin{array}{l}\text { Seuil correspondant à } \\
\text { une baisse de fécondité }\end{array}$ \\
\hline \multirow{2}{*}{ Volume du sperme $: \geq 2 \mathrm{ml}$} & $<2 \mathrm{ml}$ : hypospermie & \\
\hline & $>6 \mathrm{ml}:$ hyperspermie & \\
\hline \multicolumn{3}{|l|}{$\mathrm{pH}$ du sperme : 7,2 - 8} \\
\hline Numération des spermatozoïdes : & 0 : azoospermie & \multirow{3}{*}{$<5$ millions $/ \mathrm{ml}$} \\
\hline \multirow[t]{2}{*}{$\geq 20$ millions $/ \mathrm{ml}$} & $<20$ millions/ml : oligospermie & \\
\hline & > 200 millions/ml : polyspermie & \\
\hline Mobilité $\geq 50 \%$ & $<50 \%$ asthénospermie & 20 à $30 \%$ \\
\hline Morphologie normale $\geq 50 \%$ & $<50 \%$ : tératospermie & 30 à $40 \%$ \\
\hline Vitalité $: \geq 50 \%$ & $<50 \%$ : nécrospermie & \\
\hline Leucocytes $<1$ million/ml & $>1 \mathrm{million} / \mathrm{ml}:$ leucospermie & \\
\hline
\end{tabular}


Tableau 2: Principes de dosage des paramètres biochimiques.

\begin{tabular}{|c|c|c|}
\hline Paramètres & Principes de dosage & Valeurs normales \\
\hline$\alpha$-glucosidase & Méthode avec p-Nitrophénol glucopyranoside (Cooper et al., 1990) & 20 mU/éjaculat \\
\hline Zinc & $\begin{array}{l}\text { Réaction avec le } 2 \text { (5-Bromo-2- pyridylazo) - } 5 \text { - [- N - propyl - } \\
\text { N-(3- sulfopropyl) amino phénol]. }\end{array}$ & $2,1 \mathrm{mmol} / \mathrm{l}$ \\
\hline Calcium & Réaction avec l'ortho-crésolphtaleïne complexon & $7 \mathrm{mmol} / \mathrm{l}$ \\
\hline Magnésium & Réaction avec le calmagite en milieu alcalin & $3,6 \mathrm{mmol} / \mathrm{l}$ \\
\hline Fructose & Réaction de Selivanoff, & $5,5-27,5 \mathrm{mmol} / \mathrm{l}$ \\
\hline Glucose & Réaction de Trinder (glucose oxydase, péroxydase) & $0,39 \mathrm{mmol} / \mathrm{l}$ \\
\hline Cholestérol & Réaction de Trinder (cholestérol oxydase, péroxydase) & $2,58 \mathrm{mmol} / \mathrm{l}$ \\
\hline Triglycéride & Réaction de Trinder (oxydase, péroxydase) & - \\
\hline Protéines & Réaction du biuret & $21-66 \mathrm{~g} / 1$ \\
\hline Sodium & Photométrie de flamme & $121 \mathrm{mmol} / \mathrm{l}$ \\
\hline Potassium & Photométrie de flamme & $23 \mathrm{mmol} / \mathrm{l}$ \\
\hline
\end{tabular}




\section{RESULTATS}

\section{Caractéristiques de la population d'étude}

La population d'étude était constituée de 220 sujets âgés de 23 à 64 ans avec une moyenne de 36 ans. La majorité de la population d'étude, soit $55 \%$ a un âge compris entre 30 et 40 ans. Seuls 35 sujets, soit 15,9\% avaient un spermogramme normal contre $84,1 \%$ d'anomalies du spermogramme. La répartition des anomalies du spermogramme observées était : asthénospermie (25\%), oligoasthénospermie $(15,5 \%)$, oligo-asthénotératospermie $(13,6 \%)$, azoospermie $(10,5 \%)$, oligospermie $(10,5 \%)$ et tératospermie $(9,1 \%)$.

\section{Paramètres biochimiques du liquide séminal \\ Variation des paramètres biochimiques en fonction de l'âge}

Les données du Tableau 3 montrent une augmentation significative du glucose et du potassium dans la tranche d'âge de 40 à 50 ans et des triglycérides entre 20 et 30 ans. Par contre, la baisse de l' $\alpha$-glucosidase est significative dans la tranche d'âge de 40 à 50 ans.

Variation des paramètres biochimiques en fonction du pH et du volume de sperme

Les résultats des dosages biochimiques en fonction du $\mathrm{pH}$ (Tableau 4) mettent en évidence une baisse significative du zinc et du calcium lorsque le liquide séminal est alcalin. Dans l'hypospermie, on note une baisse de l' $\alpha$-glucosidase et une diminution significative du cholestérol, du zinc, du calcium et du potassium dans l'hyperspermie. Valeurs moyennes des paramètres biochimiques en fonction des anomalies du spermogramme

Dans le Tableau 5 est présenté une vue globale des paramètres biochimiques en fonction des anomalies du spermogramme. L' $\alpha$-glucosidase, les triglycérides, le magnésium, le zinc, le calcium, le sodium, le potassium et les protéines totales ont présenté des variations significatives. Parmi ces paramètres, le zinc a augmenté significativement dans toutes les anomalies en dehors de l'oligospermie, le magnésium est abaissé dans l'asthénospermie et les anomalies qui lui sont associées et le potassium abaissé dans l'oligospermie et l'oligo-asthénospermie. L' $\alpha$-glucosidase quant à lui, est abaissé dans l'azoospermie et l'oligozoospermie.

Corrélations $r 2$ entre les paramètres biochimiques et les caractéristiques du spermogramme

L'étude des corrélations entre les paramètres du spermogramme et les concentrations des paramètres biochimiques (Tableau 6) a permis de noter des corrélations entre le volume et les paramètres cholestérol, zinc, potassium, entre le $\mathrm{pH}$ et les concentrations de zinc et calcium, entre la mobilité et le zinc, entre la vitalité et le zinc et enfin entre $\alpha$-glucosidase et nombre de spermatozoïdes.

\section{DISCUSSION}

Caractéristiques de la population

La moyenne d'âge de la population étudiée était de 37 ans avec des extrêmes de 23 et 64 ans. La majorité, soit $55 \%$ de la population, était constituée par la tranche d'âge de 30 à 40 ans. Il s'agit d'un échantillonnage comparable à ceux de la littérature (Mona, 1984; Ouegnin et al., 1988 ; Nsembani, 1992 ; Kaba, 2002 ; Fofana, 2004). Ces études ont montré la prédominance de l'hypofertilité masculine entre 30 et 40 ans. Ceci pourrait s'expliquer par le fait que cette tranche d'âge correspond à la période où l'homme accorde beaucoup plus d'intérêt à la reproduction dans sa vie conjugale. Une autre hypothèse pourrait être que cette tranche d'âge correspond à la période d'hésitation ou de prise de conscience tardive de l'homme pour l'établissement de sa part de responsabilité dans la stérilité conjugale. 
Tableau 3 : Paramètres biochimiques de la population d'étude en fonction de l'âge.

\begin{tabular}{|c|c|c|c|c|}
\hline Paramètres & $\begin{array}{l}\text { Population totale } \\
\qquad \begin{array}{c}(\mathbf{n}=\mathbf{2 2 0}) \\
\mathrm{M} \pm \mathrm{ET}\end{array}\end{array}$ & $\begin{array}{c}20-30 \text { ans } \\
n=39 \\
M \pm E T\end{array}$ & $\begin{array}{c}\text { 30-40 ans } \\
n=121 \\
M \pm E T\end{array}$ & $\begin{array}{c}40-50 \text { ans } \\
n=60 \\
M \pm E T\end{array}$ \\
\hline$\alpha$-glucosidase & $19 \pm 5$ & $23 \pm 3$ & $20 \pm 2$ & $17 \pm 3^{*}$ \\
\hline Fructose (mmol/l) & $23,6 \pm 12$ & $23,4 \pm 12,1$ & $25,4 \pm 13,07$ & $24,04 \pm 10,82$ \\
\hline Glucose (mmol/l) & $0,8 \pm 1,83$ & $0,66 \pm 0,51$ & $0,60 \pm 0,58$ & $1,18 \pm 0,19 *$ \\
\hline Cholestérol (mmol/l) & $0,72 \pm 0,47$ & $0,78 \pm 0,61$ & $0,68 \pm 0,36$ & $0,78 \pm 0,59$ \\
\hline Triglycérides (mmol/l) & $0,98 \pm 0,98$ & $1,20 \pm 0,93 *$ & $0,89 \pm 0,68$ & $1,09 \pm 0,83$ \\
\hline Magnésium (mmol/l) & $5,26 \pm 6,92$ & $6,79 \pm 8,26$ & $5,41 \pm 7,52$ & $5,55 \pm 6,23$ \\
\hline Zinc (mmol/l) & $3,93 \pm 2,85$ & $4,20 \pm 3,03$ & $3,71 \pm 2,71$ & $4,45 \pm 3,25$ \\
\hline Calcium (mmol/l) & $3,7 \pm 2,1$ & $3,70 \pm 2,31$ & $3,42 \pm 1,82$ & $3,70 \pm 2,24$ \\
\hline Sodium (mmol/l) & $117,4 \pm 13,4$ & $119,3 \pm 18,5$ & $114,7 \pm 13,1$ & $117,8 \pm 14,5$ \\
\hline Potassium (mmol/l) & $32,58 \pm 7,7$ & $31 \pm 7$ & $32,1 \pm 7,5$ & $35 \pm 8,3 *$ \\
\hline Protéines totales (g/l) & $27,28 \pm 6,79$ & $27,69 \pm 7,72$ & $27,65 \pm 6,52$ & $27,90 \pm 6,00$ \\
\hline Albumine $(\mathrm{g} / \mathrm{l})$ & $2,51 \pm 1,21$ & $2,1 \pm 1,3$ & $2,3 \pm 1,1$ & $2,7 \pm 1,1$ \\
\hline$\alpha$ globulines (g/l) & $6,8 \pm 3,35$ & $8,2 \pm 2,3$ & $7,1 \pm 3,5$ & $7,5 \pm 3,4$ \\
\hline$\beta$ globulines $(\mathrm{g} / \mathrm{l})$ & $15,16 \pm 5,72$ & $15,9 \pm 6,3$ & $14,2 \pm 5,3$ & $15,6 \pm 5,4$ \\
\hline$\gamma$ globulines $(\mathrm{g} / \mathrm{l})$ & $2,86 \pm 1,73$ & $2,7 \pm 2,3$ & $2,8 \pm 2,4$ & $2,9 \pm 1,7$ \\
\hline
\end{tabular}


Tableau 4: Résultats des dosages biochimiques en fonction du pH et du volume.

\begin{tabular}{|c|c|c|c|c|c|}
\hline \multirow[t]{2}{*}{ Paramètres } & \multicolumn{2}{|c|}{ pH } & \multicolumn{3}{|c|}{ Volume } \\
\hline & Normal n=141 & Alcalin n=79 & Normal n=177 & $\begin{array}{c}\text { Hypospermie } \\
\text { N=36 }\end{array}$ & $\begin{array}{c}\text { Hyperspermie } \\
\text { N=7 }\end{array}$ \\
\hline$\alpha$-glucosidase(mUI/éjaculat) & $21 \pm 2$ & $19 \pm 3$ & $20 \pm 2$ & $16 \pm 2 *$ & $19 \pm 4$ \\
\hline Fructose (mmol/l) & $23,96 \pm 11,55$ & $23,1 \pm 13,07$ & $23,40 \pm 11,95$ & $22,89 \pm 10,2$ & $34,03 \pm 20,80$ \\
\hline Glucose (mmol/l) & $0,81 \pm 1,48$ & $0,8 \pm 2,33$ & $0,77 \pm 1,67$ & $1,08 \pm 2,70$ & $0,43 \pm 0,55$ \\
\hline Cholestérol (mmol/l) & $0,75 \pm 0,49$ & $0,68 \pm 0,44$ & $0,72 \pm 0,47$ & $0,84 \pm 0,4$ & $0,27 \pm 0,14^{*}$ \\
\hline Triglycérides (mmol/l) & $1,01 \pm 1,11$ & $0,92 \pm 0,69$ & $1,00 \pm 1,04$ & $0,92 \pm 0,66$ & $0,62 \pm 0,58$ \\
\hline Protéines totales $(\mathrm{g} / \mathrm{l})$ & $27,7 \pm 6,93$ & $26,51 \pm 6,55$ & $27,01 \pm 6,58$ & $28,35 \pm 7,57$ & $28,30 \pm 9,05$ \\
\hline Magnésium (mmol/1) & $5,32 \pm 7,27$ & $5,16 \pm 6,39$ & $5,19 \pm 6,72$ & $5,42 \pm 6,16$ & $7,44 \pm 14,49$ \\
\hline Zinc (mmol/l) & $4,84 \pm 2,97$ & $3,12 \pm 2,5 *$ & $3,89 \pm 2,80$ & $5,34 \pm 3,42$ & $1,74 \pm 0,98^{*}$ \\
\hline Calcium (mmol/l) & $4,05 \pm 2,14$ & $3,1 \pm 1,86 *$ & $3,72 \pm 2,06$ & $4,09 \pm 2,32$ & $1,90 \pm 1,13^{*}$ \\
\hline Sodium (mmol/l) & $116,5 \pm 12,2$ & $118 \pm 15$ & $116,81 \pm 13,80$ & $123,24 \pm 9,18$ & $116,67 \pm 11,15$ \\
\hline Potassium (mmol/l) & $33,2 \pm 7,4$ & $31,3 \pm 7,8$ & $32,62 \pm 7,33$ & $35,47 \pm 9,64$ & $23,17 \pm 4,96^{*}$ \\
\hline Protéines totales $(\mathrm{g} / \mathrm{l})$ & $27,7 \pm 6,93$ & $26,51 \pm 6,55$ & $27,01 \pm 6,58$ & $28,35 \pm 7,57$ & $28,30 \pm 9,05$ \\
\hline Albumine $\mathrm{g} / \mathrm{l}$ & $2,5 \pm 1,2$ & $2,5 \pm 1,2$ & $2,5 \pm 1,2$ & $2,8 \pm 1,5$ & $2,7 \pm 1,2$ \\
\hline$\alpha$ globulines $\mathrm{g} / \mathrm{l}$ & $6,4 \pm 3,0$ & $7,0 \pm 3,5$ & $6,8 \pm 3,3$ & $7,3 \pm 3,6$ & $4,4 \pm 4,3$ \\
\hline$\beta$ globulines $\mathrm{g} / \mathrm{l}$ & $14,8 \pm 5,6$ & $15,4 \pm 5,9$ & $15,0 \pm 5,6$ & $15,7 \pm 6,2$ & $17,3 \pm 6,6$ \\
\hline$\gamma$ globulines $\mathrm{g} / \mathrm{l}$ & $2,8 \pm 1,7$ & $2,9 \pm 1,8$ & $2,8 \pm 1,6$ & $2,6 \pm 2,0$ & $3,9 \pm 1,6$ \\
\hline
\end{tabular}


Tableau 5: Paramètres biochimiques en fonction des anomalies du spermogramme.

\begin{tabular}{|c|c|c|c|c|c|c|c|}
\hline Paramètres & $\begin{array}{c}\text { Normoospermie } \\
\quad n=35\end{array}$ & $\begin{array}{c}\text { Azoospermie } \\
n=23\end{array}$ & $\begin{array}{c}\text { Oligospermie } \\
n=23\end{array}$ & $\begin{array}{c}\text { Asthénospermie } \\
\quad n=55\end{array}$ & $\begin{array}{c}\text { Tératospermie } \\
n=20\end{array}$ & $\begin{array}{c}\text { Oligo- } \\
\text { Asthénospermie } \\
n=34\end{array}$ & $\begin{array}{c}\text { Oligo-Asthéno- } \\
\text { tératospermie } \\
(\text { OATS }) \\
n=30\end{array}$ \\
\hline & $\begin{array}{c}\text { Moyenne } \pm \text { Écart- } \\
\text { type }\end{array}$ & Moyenne \pm Écart-type & $\begin{array}{c}\text { Moyenne } \pm \text { Écart- } \\
\text { type }\end{array}$ & $\begin{array}{c}\text { Moyenne } \pm \text { Écart- } \\
\text { type }\end{array}$ & $\begin{array}{c}\text { Moyenne } \pm \text { Ecart- } \\
\text { type }\end{array}$ & $\begin{array}{c}\text { Moyenne } \pm \text { Ecart- } \\
\text { type }\end{array}$ & $\begin{array}{l}\text { Moyenne } \pm \\
\text { Écart-type }\end{array}$ \\
\hline $\begin{array}{l}\alpha \text {-glucosidase } \\
\text { (mUI/éjaculat) }\end{array}$ & $19 \pm 3$ & $14 \pm 3 *$ & $16 \pm 3 *$ & $18 \pm 3$ & $18 \pm 4$ & $19 \pm 2$ & $18 \pm 2$ \\
\hline Fructose $(\mathrm{mmol} / \mathrm{l})$ & $25,51 \pm 11,24$ & $24,40 \pm 10,65$ & $20,65 \pm 14,27$ & $22,76 \pm 12,23$ & $20,92 \pm 10,96$ & $25,19 \pm 12,93$ & $25,07 \pm 12,01$ \\
\hline Glucose (mmol/l) & $0,59 \pm 0,60$ & $0,59 \pm 0,43$ & $0,74 \pm 0,77$ & $0,94 \pm 2,15$ & $0,47 \pm 0,21$ & $1,17 \pm 3,55$ & $0,82 \pm 1,05$ \\
\hline Cholestérol (mmol/l) & $0,75 \pm 0,52$ & $0,62 \pm 0,40$ & $0,76 \pm 0,48$ & $0,72 \pm 0,37$ & $0,72 \pm 0,37$ & $0,66 \pm 0,49$ & $0,84 \pm 0,63$ \\
\hline Triglycérides (mmol/l) & $0,77 \pm 0,49$ & $0,75 \pm 0,73$ & $1,28 \pm 2,13$ & $1,11 \pm 0,79 *$ & $0,95 \pm 0,97$ & $0,94 \pm 0,70$ & $0,98 \pm 0,77$ \\
\hline Magnésium (mmol/l) & $7,24 \pm 5,22$ & $6,25 \pm 8,35$ & $5,42 \pm 7,90$ & $4,48 \pm 5,64 *$ & $5,48 \pm 5,55$ & $4,36 \pm 6,14^{*}$ & $4,49 \pm 3,63^{*}$ \\
\hline Zinc (mmol/l) & $2,38 \pm 1,55$ & $4,43 \pm 2,87^{*}$ & $3,16 \pm 2,79$ & $3,53 \pm 2,71 *$ & $4,31 \pm 3,15^{*}$ & $3,63 \pm 2,99 *$ & $6,07 \pm 2,94 *$ \\
\hline Calcium (mmol/l) & $3,62 \pm 2,49$ & $3,27 \pm 2,02$ & $3,76 \pm 2,33$ & $3,88 \pm 1,77$ & $3,83 \pm 1,85$ & $3,28 \pm 1,67$ & $4,21 \pm 2,64^{*}$ \\
\hline Sodium $(\mathrm{mmol} / \mathrm{l})$ & $117,70 \pm 13,40$ & $124,79 \pm 12,75^{*}$ & $117,41 \pm 10,77$ & $114,89 \pm 2,02$ & $119,58 \pm 13,26$ & $117,65 \pm 17,06$ & $113,64 \pm 11,70$ \\
\hline Potassium (mmol/l) & $33,7 \pm 8,1$ & $32,1 \pm 9,4$ & $28,1 \pm 8,1^{*}$ & $34,1 \pm 7,2$ & $33,3 \pm 7,2$ & $30,3 \pm 5,5^{*}$ & $34,3 \pm 7,9$ \\
\hline Protéines totales $(\mathrm{g} / \mathrm{l})$ & $28,2 \pm 6,6$ & $26,4 \pm 6,1$ & $24,1 \pm 6,7^{*}$ & $26,82 \pm 6,58$ & $27,04 \pm 4,30$ & $28,77 \pm 7,56$ & $28,54 \pm 7,85$ \\
\hline Albumine $(\mathrm{g} / \mathrm{l})$ & $2,59 \pm 1,35$ & $2,25 \pm 0,92$ & $2,69 \pm 1,59$ & $2,59 \pm 1,35$ & $2,41 \pm 0,71$ & $2,31 \pm 1,01$ & $2,60 \pm 1,19$ \\
\hline$\alpha$ globulines $(\mathrm{g} / \mathrm{l})$ & $7,61 \pm 4,52$ & $7,27 \pm 3,79$ & $5,48 \pm 2,16$ & $7,14 \pm 2,98$ & $6,75 \pm 3,80$ & $6,04 \pm 2,60$ & $6,73 \pm 3,01$ \\
\hline$\beta$ globulines $(\mathrm{g} / \mathrm{l})$ & $15,29 \pm 6,50$ & $13,62 \pm 4,52$ & $13,31 \pm 5,12$ & $14,51 \pm 5,30$ & $15,33 \pm 4,67$ & $17,29 \pm 6,06$ & $16,27 \pm 6,55$ \\
\hline$\gamma$ globulines $(\mathrm{g} / \mathrm{l})$ & $2,73 \pm 1,52$ & $3,26 \pm 2,43$ & $2,45 \pm 1,11$ & $2,60 \pm 1,58$ & $2,71 \pm 1,30$ & $3,41 \pm 2,10$ & $2,94 \pm 1,73$ \\
\hline
\end{tabular}


J. SAKANDE et al. / Int. J. Biol. Chem. Sci. 6(3): 1167-1178, 2012

Tableau 6: Coéfficient de corrélation entre les paramètres biochimiques et les caractéristiques du spermogramme.

\begin{tabular}{lcccccc}
\hline \multirow{2}{*}{ Paramètres biochimiques } & \multicolumn{5}{c}{ Paramètres du spermogramme } \\
\cline { 2 - 6 } & Age & Volume & pH & Mobilité & Vitalité & Nombre de spermatozoïdes /mm $^{\mathbf{3}}$ \\
\hline Fructose & 0,07 & 0,05 & 0,02 & 0,09 & 0,01 & 0,09 \\
Glucose & 0,09 & 0,09 & 0,04 & 0,07 & 0,03 & 0,03 \\
Cholestérol & 0,01 & $0,61^{*}$ & 0,12 & 0,01 & 0,01 & 0,03 \\
Triglycérides & 0,001 & 0,03 & 0,03 & 0,02 & 0,05 & 0,02 \\
Protéines Totales & 0,05 & 0,09 & 0,05 & 0,09 & 0,05 & 0,01 \\
Magnésium & 0,02 & 0,001 & 0,01 & 0,09 & 0,13 & 0,01 \\
Zinc & 0,06 & $0,71^{*}$ & $0,64^{*}$ & $0,51^{*}$ & $0,80^{*}$ & 0,04 \\
Calcium & 0,01 & 0,12 & 0,28 & 0,04 & 0,002 & 0,05 \\
Sodium & 0,09 & 0,07 & 0,003 & 0,02 & 0,04 & 0,08 \\
Potassium & 0,06 & $0,67^{*}$ & 0,19 & 0,01 & 0,02 & 0,07 \\
$\alpha$-glucosidase & 0,07 & 0,15 & 0,10 & 0,01 & 0,17 & $0,78^{*}$ \\
\hline
\end{tabular}

* Corrélations significatives $(\mathrm{p}<0,05)$ 


\section{Les anomalies du spermogramme}

L'étude a montré que $84,1 \%$ de la population consultant pour hypofertilité présente une anomalie du spermogramme. Ce résultat confirme ceux de Kaba (2002) qui a rapporté dans sa série $95,8 \%$ d'anomalies de spermogrammes réalisés dans un laboratoire de la ville de Ouagadougou. Fofana (2004) a également rapporté $68,6 \%$ d'éjaculats présentant des anomalies à Abidjan. Ces résultats démontrent la place prépondérante des anomalies du sperme dans l'étiologie de l'hypofertilité masculine. La répartition des anomalies du spermogramme observées dans notre étude se rapproche de celles de la plupart des auteurs qui placent l'asthénospermie au premier rang des anomalies du spermogramme chez les patients consultant pour hypofertilité en Afrique (Adendji et Olayemi 2003 ; Diafouka et Gbassi, 2009 ; Ugboaja et al., 2010). Il est à noter que l'azoospermie occupe également une part importante dans l'hypofertilité avec $10,5 \%$ de fréquence dans notre série. Cette valeur est proche de celle de Nguema (1986) au Gabon avec 13,4\%, Diawara (1995) en Côte d'Ivoire (12,3\%) et Locko (1989) au Congo Brazzaville (8\%). Kaba (2002) quant à lui dans sa série à Ouagadougou a rapporté une fréquence de l'azoospermie plus élevée, soit $19,3 \%$.

\section{Les résultats biochimiques}

Variation des paramètres biochimiques avec l'âge

Le dosage des paramètres biochimiques a mis en évidence des perturbations essentiellement dans la tranche d'âge de 40 à 50 ans. Il s'agit d'une baisse significative de l' $\alpha$-glucosidase et au contraire une augmentation du glucose et du potassium. Ces perturbations pourraient être en rapport avec la baisse de la qualité du sperme avec l'âge. La baisse de l' $\alpha$-glucosidase est habituellement observée dans les obstructions épididymaires, du déférent et des canaux éjaculateurs. Dans la tranche d'âge de 20 à 30 ans nous avons observé une augmentation significative des triglycérides. La même observation a été rapportée par Beer (2009) dans une étude expérimentale chez les jeunes taureaux. Cet auteur avait postulé que l'augmentation des triglycérides pourrait être utilisée comme un marqueur biochimique prédictif de la qualité du sperme. L'augmentation significative des triglycérides dans la tranche jeune de notre étude pourrait présenter un intérêt et des travaux futurs sont nécessaires pour le confirmer.

Variation des paramètres biochimiques en fonction du pH et du volume du sperme

Considérant le $\mathrm{pH}$ du sperme, l'étude a montré que $65 \%$ de la population avaient un liquide séminal basique. Ces résultats sont très différents de ceux de Kaba (2002) qui ne retrouvait que 32,3\% d'échantillons basiques. Parmi les constantes biochimiques, seules le zinc et le calcium sont significativement diminués dans les liquides séminaux alcalins et ceci pourrait s'expliquer par le fait que ces ions sont des composants de la sécrétion prostatique acide. Cette alcalinisation du sperme pourrait donc être consécutive à une diminution de la sécrétion prostatique riche en phosphatase acide. Cette diminution des phosphatases acides est observée dans les infections prostatiques ou les obstructions prostatiques. De même, la diminution du zinc observée est un excellent marqueur de prostatite généralement liée aux infections (Bobak et al., 2009 ; Rusz et al., 2012 ). Quant à la mesure du volume spermatique nous avons observé $19 \%$ de perturbations dont $16 \%$ d'hypospermie et 3\% d'hyperspermie. Ces valeurs se rapprochent de celles de Kaba (2002) qui trouvait respectivement $18,8 \%$ d'hypospermie et $4,8 \%$ d'hyperspermie. Quant à Ravalomanana (2001), il a trouvé $21,65 \%$ d'hypospermie et $2,85 \%$ d'hyperspermie. Cet auteur a rapporté une corrélation entre l'hypospermie et l'urétrite. D'autres auteurs ont rapporté une baisse significative de l'a-glucosidase dans l'hypospermie au cours de l'occlusion épididymaire ou déférentielle (Zopfgen et al., 2000 ; Morretti et al., 2009 ; Said et al., 2009). $\mathrm{Au}$ cours de l'hyperspermie, nous avons observé une baisse des valeurs du cholestérol, $\mathrm{du}$ zinc, $\mathrm{du}$ calcium et du potassium. L'hyperspermie est quelque fois en rapport avec un syndrome inflammatoire ou infectieux.

Variation des paramètres biochimiques avec les anomalies du spermogramme

La comparaison des paramètres biochimiques en fonction des anomalies du 
spermogramme a montré de nombreuses perturbations. L' $\alpha$-glucosidase est diminué dans l'azoospermie et l'oligospermie, ceci est en rapport avec l'étiologie occlusive des voies génitales. Le zinc est augmenté dans toutes les anomalies sauf dans l'oligospermie. Le magnésium est diminué dans les anomalies touchant la mobilité, notamment l'asthénospermie et les anomalies qui lui sont associées. Le rôle indispensable de cet ion dans la mobilité cellulaire pourrait justifier les nombreuses anomalies du sperme concomitamment observées avec sa baisse dans le liquide séminal (Kumar et al., 2006). Le sodium est augmenté uniquement dans l'azoospermie et les triglycérides, dans l'asthénospermie. Ceci est en accord avec les résultats trouvés par Minal (2006).

\section{Conclusion}

$\mathrm{Au}$ total, en dehors des paramètres classiques de la biochimie séminale, la détermination du profil électrophorétique des protéines du liquide séminal n'a pas apporté un intérêt notable dans l'étude des différentes anomalies du spermogramme. Cependant, l'augmentation du sodium dans l'azoospermie, la diminution du magnésium et l'augmentation des triglycérides dans l'asthénospermie et la diminution du potassium dans l'oligospermie présenteraient un intérêt s'ils étaient confirmés par d'autres travaux. En effet, ces paramètres de réalisation courante dans la plupart des laboratoires africains pourraient être utiles dans l'exploration de l'hypofertilité masculine.

\section{REFERENCES}

Adendji RA, Olayemi O. 2003. Pattern of semen analysis of male partners of infertile couples at the University college Hospital, Ibadan. W. A. J. O., 22(3): 243245.

AFSSAPS. 2004. Recommandations de bonne pratique : Les médicaments inducteurs de l'ovulation. Rapport, Agence Française de Sécurité Sanitaire des Produits de Santé (AFSSAPS), Paris.

Beaudeux JL, Durand G. 2011. Biochimie Médicale, Marquers Actuels et Perspectives ( $2^{\mathrm{e}}$ edn). Lavoisier: Paris.
Beer-Ljubic B, Aladrovic J, Marenjac TS, Laskaj R, Majic-Balic I, Milinkovic-Tur S. 2009. Cholesterol concentration in seminal plasma as a predictive tool for quality semen evaluation. Theriogenology, 72(8): 1132-1140.

Bobak L, Rosocha J, Lehocka L, Halagovec A, Hrivnak M, Kraus V, Petrovicova J. 2009. Correlations between hyperviscosity of the ejaculate and physicalmorphological and biochemical parameters. Ceska Gynekol, 74(3): 219224.

Delattre J, Durand G, Jardillier JC. 2003. Biochimie Pathologique - Aspects Moléculaires et Cellulaires. Lavoisier: Paris.

Diafouka F, Gbassi GK. 2009. Deficiency of $\alpha$-tocopherol in seminal fluid as a probable factor in low fertility in Cote d'Ivoire. Afr. J. Reprod. Health., 13(3): 123-125.

Diawara S. 1995. Evaluation fonctionnelle du testicule exocrine dans l'infertilité masculine par le spermogramme et le profil électrophorétique des protéines du plasma séminal : à propos de 65 éjaculats. Thèse de Pharmacie, Université d'Abidjan, p. 144.

EDSBF (Enquête démographique et de Santé du Burkina Faso). 2003. Rapport EDSBF Min. Santé, Ouagadougou.

Fofana YB. 2004. Profils spermiologiques et concentration séminale de l'haptoglobine chez des patients consultant pour hypofertilité conjugale à composante masculine. A propos de 70 cas. Thèse de pharmacie, Université d'Abidjan, p.80.

Kaba L. 2002. Prévalence des anomalies du spermogramme au laboratoire Ste Elisabeth de Ouagadougou. Mémoire Licence spécialisée en biologie, Université de Ouagadougou, p. 45.

Kumar N, Verma RP, Singh LP, Varshney VP, Dass RS. 2006. Effect of different levels and sources of zinc supplementation on quantitative and qualitative semen attributes and serum testosterone level in crossbred cattle (Bos indicus x Bos taurus). Reprod. Nutr. Dev., 46(6): 663-675. 
Locko C, Mafouta G, Malonga F, Diafouka F. 1989. La stérilité masculine à Brazzaville (à propos de 100 cas). Médecine d'Afrique Noire, 36(4) : 326 - 330.

Minal M, Sathwara NG. 2006. Seminal plasma zinc concentration and glycosidase activity with respect to semen quality. Biological Trace Element Research, 110: 15-25.

Mona H. 1984. Contribution à l'étude de la part de l'infertilité masculine dans la stérilité conjugale : à propos de 258 cas. Thèse médecine, Université d'Abidjan, p.92.

Morretti E, Cosci I, Spreafico A, Serchi T, Cuppone AM, Collodel G. 2009. Semen characteristics and inflammation mediators in infertile men with different clinical diagnosis. Int. J. Androl., 32(6): 637-646.

N'Guema E, Mavoungou D, Gass R, Roth M C, Jouannet P. 1986. Comparaison des paramètres spermiologiques d'un groupe fécond et d'un groupe infécond. ChirfGabon, 2: 28-33.

Nsembani E. 1992. Profils spermiologiques dans la stérilité masculine au CHU Marien N'Gouabi. Thèse médecine, Université de Brazzaville, p. 364.

Ouegnin GA, Sess D, Dossou M, Chenal H, Khalaf N, Tanoe JM, Ehouman A, Yao DC. 1988. Approche diagnostic de l'infertilité masculine dans le service d'urologie du CHU de Cocody. Soci. Med., 4: 18-27.
Ravolamanana R, Randaoharison PG, Ralaiavy HA, Debry JM. 2001. Approche étiologique des stérilités du couple à Mahajanga. Archives Institut Pasteur Madagascar, 67: 68-73.

Rusz A, Pilatz A, Wagenlehner F, Linn T, Diemer T, Schuppe HC, Lohmeyer J, Hossain H, Weidner W. 2012. Influence of urogenital infections an inflammation on semen quality and male fertility. World J. Urol., 30(1): 23-30.

Said L, Gleraud-Denis I, Carreau S, Saad A. 2009. Relation between semen quality and seminal plasma components: $\alpha$ glucosidase, fructose and citrate in infertile men compared with a normospermic population of Tunisian men. Andrologia, 41(3): 150-6.

Ugboaja JO, Monago EN, Obiechina NJ. 2010. Pattern of semen fluid abnormalities in male partners of infertile couples in Southeastern Nigeria. Niger. J. Med., 19(3): 286-8.

WHO. 2010. Laboratory Manual for the Examination and Processing of Human Semen ( $4^{\text {th }}$ edn). WHO: Geneva.

Zopfgen A, Prem F, Sudhoff F, Jung K, Leek S, Loening SA, Sinha P. 2000. Relationship between semen quality and seminal plasma components carnitin, $\alpha$ glucosidase, fructose, citrate and granulocyte elastase in infertile men compared with a normal population. Oxford Journal Medecine Human Reproduction, 15(4): 840-845. 